\title{
First-Principles Study of Structural, Elastic and Mechanical Properties of Zinc-Blende Boron Nitride (B3-BN)
}

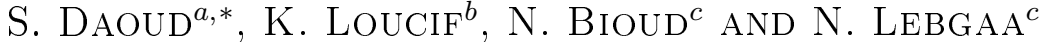 \\ ${ }^{a}$ Institut des Sciences et Technologies, Centre Universitaire de Bordj Bou Arreridj, Algeria \\ ${ }^{b}$ Laboratoire des matériaux non métalliques, Université Ferhat Abbas, Sétif, Algeria \\ ${ }^{c}$ Laboratoire d'optoélectronique \& Composants, Université Ferhat Abbes, Sétif, Algeria
}

(Received May 19, 2011; revised version January 21, 2012; in final form February 21, 2012)

\begin{abstract}
First principles study of structural, elastic properties and anisotropy effect on the mechanical parameters of the zinc-blende boron nitride has been performed using the pseudopotential plane wave method based on density functional theory with the Teter and Pade exchange-correlation functional of the local density approximation. The equilibrium lattice constant, molecular and crystal densities, bond length, the independent elastic constants, bulk modulus and its pressure derivatives, compressibility, shear modulus, internal strain parameter, isotropy factor, compliance constants, the Debye temperature, Young's modulus, Poisson's ratio, the Lamé constants and sound velocity for directions within the important crystallographic planes of this compound are obtained and analyzed in comparison with the available theoretical data reported in the literature.
\end{abstract}

PACS: 45.10.-b, 62.20.D-, 61.66.-f, 62.20.de, 81.40.Jj, 31.15.E-

\section{Introduction}

There is considerable interest in the study of properties of zinc-blende boron nitride compound. From a technological point of view this material has useful physical properties, like extreme hardness, high melting point, and interesting dielectric and thermal characteristics $[1,2]$.

The boron nitride $(\mathrm{BN})$ is normally found in the hexagonal phase [3], the zinc-blende structure was synthesized [4] in 1957. Like diamond, BN is known to be a very hard material [5].

Many papers [1, 2, 4, 6-24] have studied structural, electronic and elastic properties of this compound, but only a few of these papers [17] dealt with theoretical aspect have been devoted to the study of the anisotropy effect on their mechanical parameters.

In order to fully take advantage of the properties of boron nitride for eventual technological applications, a theoretical investigation of the sound velocity, and anisotropy effect on the mechanical parameters, is necessary. First-principles calculations offer one of the most powerful tools for carrying out theoretical studies of these properties. In the present work, we report first-principles study of structural and elastic properties, sound velocity, the Debye temperature, and anisotropy effect on the mechanical parameters for boron nitride compound in its zinc-blende phase. This is done using the state of the art pseudopotential plane wave method in the framework of the density functional theory within the local density approximation.

The paper is organized as follows: in Sect. 2, we briefly describe the computational techniques used in this work.

Results and discussions of the structural and elastic

* corresponding author; e-mail: salah_daoud07@yahoo.fr properties, sound velocity, the Debye temperature, and anisotropy effect on the mechanical parameters are presented in Sect. 3. Finally, conclusions and remarks are given in Sect. 4.

\section{Computational methods}

The first-principles calculations were performed by employing pseudopotential plane-waves (PP-PW) approach based on the density functional theory (DFT) [25] and implemented in the ABINIT code [26]. ABINIT computer code is a common project of the Universite Catholique de Louvain, Corning Incorporated, and other contributors. We used the Teter and Pade (fitting of PW92 data) parameterization [27] for local density approximation (LDA). Only the outermost electrons of each atom were explicitly considered in the calculation. The effect of the inner electrons and the nucleus (the frozen core) was described within a pseudopotential scheme. We used the Trouiller-Martins scheme [28] to generate the norm-conserving nonlocal pseudopotentials, which results in highly transferable and optimally smooth pseudopotentials. A plane-wave basis set was used to solve the Kohn-Sham equations in the pseudopotential implementation of the DFT-LDA. The major advantages of this approach are: the ease of computing forces and stresses; good convergence control with respect to all employed computational parameters; favourable scaling with number of atoms in the system and the ability to make cheaper calculations by neglecting core electrons [29].

The Brillouin zone integrations were replaced by discrete summations over a special set of $k$-points, using the standard $k$-point technique of Monkhorst and Pack [30] where the $k$-point mesh used is $(4 \times 4 \times 4)$. The plane-wave energy cut-off to expand the wave functions is set to be $70 \mathrm{Ha}$. Accurate convergence tests show that with these parameters relative energy converged to better than $10^{-5} \mathrm{eV} /$ atom. 


\section{Results and discussion}

\subsection{Structural properties}

3.1.1. Equilibrium lattice constant, bulk modules $B_{0}$ and its pressure derivatives $B_{0}^{\prime}$

After having determined the kinetic energy cut-off and the number of special $k$-points which gives the best convergence possible of total energy, they are used to the calculus of total energy for various values of the lattice constant. Energies were calculated for various values of the lattice constant, the different values obtained are then presented as function of the unit cell volume.

From the total energy presented as function of the lattice volume, one can deduce the static structural properties such as the equilibrium lattice constant from the volume which gives the minimum energy, bulk modulus $B_{0}$ and its pressure derivatives $B_{0}^{\prime}$.

This can be done by fitting the values of total energy as a function of the unit cell volume to the Murnaghan equation [31] given by

$$
\begin{aligned}
& E(V)-E\left(V_{0}\right)=\frac{B_{0} V}{B_{0}^{\prime}}\left[\frac{\left(V_{0} / V\right)^{\mathrm{B}_{0}^{\prime}}}{B_{0}^{\prime}-1}+1\right]-\frac{B_{0} V_{0}}{B_{0}^{\prime}-1}, \\
& B_{0}=V \frac{\partial^{2} E}{\partial V^{2}}=\frac{4}{9 a} \frac{\partial^{2} E}{\partial a^{2}},
\end{aligned}
$$

where $B_{0}$ is the bulk modulus given by the relation (2) at $P=0, V_{0}$ is the equilibrium volume, $E\left(V_{0}\right)$ is the energy in equilibrium volume, $B_{0}^{\prime}$ is the pressure derivative of the bulk modulus and $a$ is the lattice constant.

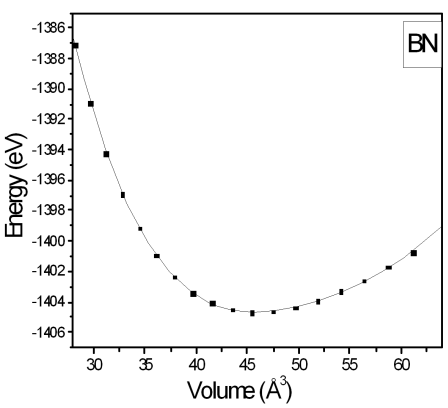

Fig. 1. Evolution of the total energy as a function of the unit cell volume of the (B3) BN.

Figure 1 shows the evolution of total energy as function of the unit cell volume of the (B3) BN. The equilibrium lattice parameter is computed also from the structural optimization, using the Broyden-FletcherGoldfarb-Shanno (BFGS) [32] minimization.

The results for: lattice parameter $a_{0}$ obtained from the fit of the Murnaghan equation and from the BFGS technique, bulk modulus $B_{0}$, and its pressure derivative $B_{0}^{\prime}$ are reported in Table I and compared with the available experimental $[1,6-9,16]$ and theoretical data $[2,4$, $10-15]$.

TABLE I

Some physical parameters of (B3) BN at zero-pressure in comparison with experimental $[1,6-9,16,18-22]$ and theoretical

\begin{tabular}{|c|c|}
\hline Parameter & \\
\hline lattice constant $a_{0}[\AA]$ & $3.593^{a}, 3.580^{b}$ \\
\hline experimental & $3.615 \pm 0.001[4]$ at $300 \mathrm{~K}, 3.615[1,6,7], 3.616[8], 3.6155 \pm 0.0002[9]$ at $300 \mathrm{~K}$ \\
\hline calculated & $3.575[10], 3.576[11], 3.62[12]$ LMTO, $3.606[2,13], 3.623[14,15]$ \\
\hline bulk modulus $B_{0}[$ Mbar $]$ & $3.76^{a}, 4.16^{c}$ \\
\hline experimental & $3.69[6], 3.72[16]$, \\
\hline calculated & $3.86[10], 3.97[11], 3.78[12]$ LMTO, $3.67[2,13], 3.65[14], 3.68[15]$ \\
\hline pressure derivatives of the bulk modulus $B_{0}^{\prime}$ & 3.86 \\
\hline experimental & $4.00[6]$ \\
\hline calculated & $3.60[11], 34[12]$ LMTO, $3.94[14], 3.32[15]$ \\
\hline compressibility $\beta\left[\mathrm{Mbar}^{-1}\right]$ & $0.266^{a}, 0.240^{c}$ \\
\hline molecular density $d_{\mathrm{M}}\left[10^{22} \mathrm{~cm}^{-3}\right]$ & $8.7179^{b}, 8.4636[17]$ \\
\hline crystal density $g\left[\mathrm{~g} / \mathrm{cm}^{3}\right]$ & $3.5928^{b}, 3.4863[8], 3.4880[17]$ at $300 \mathrm{~K}$ \\
\hline bond length $d$ (cation-anion) $[\AA]$ & $1.5501^{b}, 1.5656[17]$ at $300 \mathrm{~K}$ \\
\hline bond length $d$ (cation-cation) $[\AA]$ & $2.5314^{b}, 2.5565[17]$ at $300 \mathrm{~K}$ \\
\hline$C_{11}[\mathrm{Mbar}]$ & $8.10^{d}, 8.789^{e}$ \\
\hline experimental & $7.80[18], 8.37[19], 8.28[20], 8.12[21], 8.19[22]$ \\
\hline calculated & $8.44[10], 8.20[23], 8.18[24]$ \\
\hline$C_{12}[\mathrm{Mbar}]$ & $1.87^{d}, 1.696^{e}$ \\
\hline experimental & $2.68[18], 1.82[19], 2.11[20], 1.82[21], 1.95[22]$ \\
\hline calculated & $1.90[10], 1.90[23], 1.79[24]$ \\
\hline$C_{44}[\mathrm{Mbar}]$ & $4.83^{d}, 5.236^{e}$ \\
\hline experimental & $3.78[18], 4.93[19], 4.79[20], 4.64[21], 4.75[22]$ \\
\hline calculated & $4.83[10], 4.80[23], 4.70[24]$ \\
\hline shear modulus $G$ [Mbar] & $3.12,3.78[16], 3.19[24]$ \\
\hline internal parameter $\xi$ & $0.12^{d}, 0.39^{f}, 0.11[23]$ \\
\hline isotropy factor $A$ & $0.811,0.656[17]$ \\
\hline compliance constants $S_{i j}\left[\mathrm{Mbar}^{-1}\right]$ & $S_{11} 0.135,0.134[17],-S_{12} 0.025,0.025[17], S_{44} 0.212,0.208[17]$ \\
\hline
\end{tabular}
$[2,4,10-15,17,23,24]$ values.

${ }^{a}$ from the relation of Eq. (1), ${ }^{b}$ from the BFGS technique, ${ }^{c}$ from the relation of Eq. (8),

${ }^{d}$ from the stress-strain relation of Eqs. (5) and (6), ${ }^{e}$ from the relation of Eq. (7), ${ }^{f}$ from the relation of Eq. (9) 
As can be seen, our calculated equilibrium lattice parameter $a_{0}$ in two cases is in excellent agreement with the experimental data and previous calculations: the calculated lattice constant deviates from the measured $[1,6,7]$ and the calculated $[2,13]$ ones within $0.6 \%$ and $0.36 \%$, respectively. The bulk modulus $B_{0}$ is also in good agreement with the available experimental and theoretical data, it is deviates from the measured [16] and the calculated [12] ones within $1.08 \%$ and $0.53 \%$, respectively. The above results also show that the computational methods and parameters used in this paper are reasonable.

\subsubsection{Molecular and crystal densities and near-neighbor} and nearest-neighbors distances

The crystal density $g$ is one of the simplest and most important material parameters, and is related to the atomic arrangement and corresponding electron density map. Group IIIA atoms have three electrons with an $s^{2} p^{1}$ configuration outside a core of closed shells, and group VA atoms have five electrons in a $s^{2} p^{3}$ configuration. The IIIA and VA atoms have, therefore, an average of four valence electrons per atom available for binding.

For such a covalent bonding each $\mathrm{V}$ atom donates an electron to a III atom, so that $\mathrm{V}^{+}$and $\mathrm{III}^{-}$ions are formed, each with four valence electrons. An ionic bond is due to the Coulomb attraction between the excess positive and negative charges on ions formed by transfer of electrons from the metallic to the nonmetallic atom. The bonds in most III-V compounds are not adequately described by any of these extreme types, but have intermediate characteristics intermediate between those usually associated with the terms covalent and ionic.

There are four molecules in a unit cell of the zinc-blende lattice. If an accurate lattice constant is available, the calculation of $g$ gives in principle a good, reliable value.

For BN each B $(\mathrm{N})$ has four nearest neighbors of B $(\mathrm{N})$ at a distance of $\sqrt{3} a_{0} / 4$ at the corners of a regular tetrahedron. The spacing between the bond length B-B (or $\mathrm{N}-\mathrm{N}$ ) atoms is equal to $a_{0} / \sqrt{2}$. The calculated molecular and crystal densities, and the bond length distances from the lattice constant obtained from the BFGS technique for (B3) BN at zero-pressure are reported also in Table I and compared with the available experimental [8] and theoretical data [17] obtained at ambient temperature (at $300 \mathrm{~K})$.

\subsubsection{Hydrostatic pressure effect}

In order to further validate the reliability and accuracy of our calculated structural properties for (B3) BN, the calculated unit cell volumes under a series of applied hydrostatic pressures (in the 0 to 4 Mbar range with the step of 0.5 Mbar) were used to construct the $P-V$ data set (Fig. 2), which was subsequently fitted to a third-order by the Birch-Murnaghan equation [33]:

$$
P(V)=\frac{3}{2} B_{0}\left[\left(\frac{V_{0}}{V}\right)^{\frac{7}{3}}-\left(\frac{V_{0}}{V}\right)^{\frac{5}{3}}\right]
$$

$$
\times\left\{1+\frac{3}{4}\left(4-B_{0}^{\prime}\right)\left[\left(\frac{V_{0}}{V}\right)^{\frac{2}{3}}-1\right]\right\},
$$

where $B_{0}$ is the bulk modulus, $V_{0}$ is the volume of unit cell, is fixed at the value determined from the zero-pressure data, $\mathrm{V}$ is the volume of unit cell at $P \neq 0$ and $B_{0}^{\prime}$ is its pressure derivative at zero pressure.

The continuity in pressure-volume phase diagram indicates the absence of the structural phase transition from zinc-blende (B3) to rock salt (B1) up to pressure of 4 Mbar.

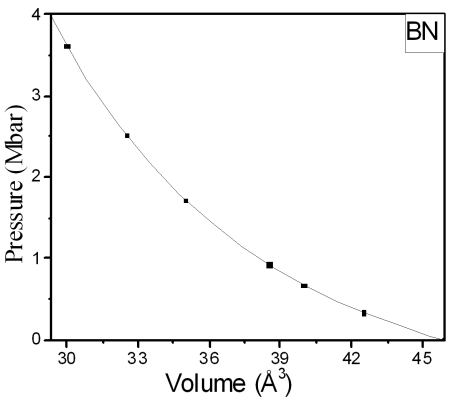

Fig. 2. $P-V$ relation for (B3) BN compound. The solid lines are given by the Birch-Murnaghan equation.

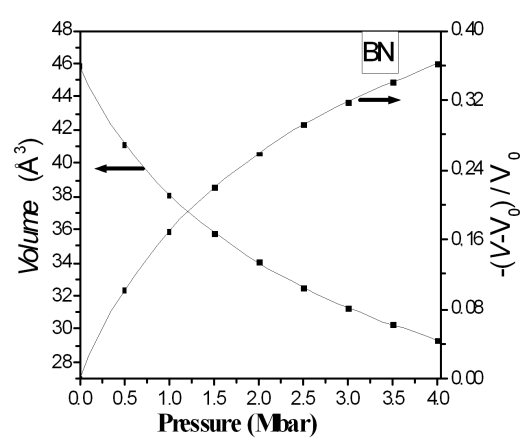

Fig. 3. Pressure dependence of the volume and $-(V-$ $\left.V_{0}\right) / V_{0}$ of (B3) BN compound.

The available theoretical data reported in the literature of this parameter are respectively: 8.50 Mbar [12], 11.1 Mbar [13], 5.5 Mbar [15], and 6.24 Mbar [34]. These values confirm also our result.

In Fig. 3 we plot the variation of the volume $V$ and the relative changes of the volume $-\left(V-V_{0}\right) / V_{0}$ versus applied hydrostatic pressure $(p)$.

\subsection{Elastic properties}

\subsubsection{Elastic stiffness constants}

The elastic constants are of interest because of the insight they give into the nature of the binding forces in solids, and they are also of importance for the thermal properties of solids. The number of independent elastic constants is usually reduced if the crystal possesses 
symmetry elements, and in the important case of cubic crystals there are only three independent stiffness constants. The array of values of the elastic stiffness constant is therefore reduced for a cubic crystal to the matrix $[C][35]$ :

$$
[C]=\left[\begin{array}{cccccc}
C_{11} & C_{12} & C_{12} & 0 & 0 & 0 \\
C_{12} & C_{11} & C_{12} & 0 & 0 & 0 \\
C_{12} & C_{12} & C_{11} & 0 & 0 & 0 \\
0 & 0 & 0 & C_{44} & 0 & 0 \\
0 & 0 & 0 & 0 & C_{44} & 0 \\
0 & 0 & 0 & 0 & 0 & C_{44}
\end{array}\right] .
$$

In a cubic lattice, three independent elastic constants $C_{11}, C_{12}$, and $C_{44}$ are determined by employing suitable lattice distortions. Following the work of Nielsen and Martin [36], we determine these constants. Values for $C_{11}$ and $C_{12}$ can be found from the stress-strain relation with the application of an $\varepsilon_{1}$ strain. This strain scales the $x$-dimensions by $\left(1+\varepsilon_{1}\right)$ while maintaining constant $y$ - and $z$-dimensions. For small strain, the harmonic approximation defines the relations as:

$$
\begin{aligned}
& C_{11}=\sigma_{1} / \varepsilon_{1}, \\
& C_{12}=\sigma_{2} / \varepsilon_{1},
\end{aligned}
$$

where $\sigma_{i}(i=1,2)$ represents the stress.

From the following stress-strain relation [36]:

$$
\sigma_{4}=\left[C_{44}^{0}-\Omega^{-1} \Phi\left(\xi a_{0} / 4\right)^{2}\right] \varepsilon_{4}=C_{44} \varepsilon_{4} .
$$

One can obtain the elastic constant $C_{44}$. In Eq. (6), $C_{0}^{44}$ denotes the elastic constant in absence of internal displacements $u, \Omega$ is the volume of the unstrained unit cell, $\Phi$ is the force constant, and $\xi$ is the internal strain parameter. With two independent calculations, setting $\varepsilon_{4}=0$ and a small relative displacement $u$ and then with small $\varepsilon_{4}$ and $u=0$, we can determine $C_{44}$ and $\xi$; details about this method can be found in Ref. [36]. The calculations were performed with $\varepsilon_{1}= \pm 0.002$ in the direction (100) to determine $C_{11}$ and $C_{12}$ and with $\varepsilon_{4}= \pm 0.004$ and $u= \pm 0.002 \sqrt{3} a_{0}$ in the direction (111) to find $C_{44}$ and $\xi$.

The obtained elastic stiffness constants $C_{11}, C_{12}, C_{44}$ and the internal strain parameter $\xi$ of (B3) BN are listed and compared with the experimental [18-22] and other theoretical [10, 23, 24] data in Table I.

The requirement of mechanical stability in a cubic crystal leads to the following restrictions on the elastic constants, $C_{11}-C_{12}>0, C_{11}>0, C_{44}>0, C_{11}+2 C_{12}>0$. The elastic constants in Table I obey these stability conditions, including the fact that $C_{12}$ must be smaller than $C_{11}$. Our calculated elastic constants also obey the cubic stability conditions, meaning that $C_{12}<B<C_{11}$. The elastic stiffness constant $C_{i j}$ versus lattice constant $a$ ( $a$ is given in $\AA$ and $C_{i j}$ in $10^{10} \mathrm{~Pa}$ ) for some cubic group IV, III-V and II-VI semiconductors can be given by [37]:

$$
\begin{aligned}
& \ln C_{11}=-4.59 \ln a+10.33, \\
& \ln C_{12}=-2.54 \ln a+6.07, \\
& \ln C_{44}=-5.20 \ln a+10.59 .
\end{aligned}
$$

For cubic crystals the bulk modulus $B_{0}$ and the compressibility $\beta$ are related to elastic constants by [38]:

$$
B_{0}=\frac{1}{\beta}=\left(C_{11}+2 C_{12}\right) / 3 \text {. }
$$

As can be seen, also that our calculated bulk modulus $B_{0}$ obtained from Eq. (8) is also in good agreement with the experimental data and previous calculations, it deviates from the measured [16] and the calculated [15] ones within $1.07 \%$ and $2.17 \%$, respectively.

For diamond and zinc-blende crystals the internal strain parameter $\xi$ and the elastic constants are also related by [39]:

$$
\xi=\frac{C_{11}+8 C_{12}}{7 C_{11}+2 C_{12}} .
$$

The shear modulus $G$ and the isotropy factor $A$ in a cubic crystal are respectively defined as [37]:

$$
\begin{aligned}
& G=\left(C_{11}-C_{12}\right) / 2, \\
& A=\left(C_{11}-C_{12}\right) / 2 C_{44} .
\end{aligned}
$$

The obtained values of the elastic stiffness constants $C_{i j}$, the internal strain parameter $\xi$, the shear modulus $G$ and the isotropy factor $A$ of (B3) BN are also listed and compared with other theoretical and experimental data in Table I.

From the data of Table I it can be seen that our calculated elastic stiffness constants $C_{11}, C_{12}$, and $C_{44}$ are in excellent agreement with the experimental and other theoretical data, they are deviating from the measured [21] respectively within $0.24 \%, 2.74 \%$ for $C_{11}$ and $C_{12}$ and from the measured [18] with $1.58 \%$ for $C_{44}$.

\subsubsection{Compliance constants}

The macroscopic theory of the elastic properties of solids is described in detail in tensor notation by Nye [40]. The elastic compliance tensor $[S]$, which has the same form as $[C]$, is connected reciprocally with the tensor $[C]$ through Hooke's relation. Explicit equations for the component $S_{i j}$ in terms of $C_{i j}$ can be given by

$$
\begin{aligned}
& S_{11}=\left(C_{11}+C_{12}\right) /\left[\left(C_{11}-C_{12}\right)\left(C_{11}+2 C_{12}\right)\right], \\
& S_{12}=\left(-C_{12}\right) /\left[\left(C_{11}-C_{12}\right)\left(C_{11}+2 C_{12}\right)\right], \\
& S_{44}=1 / C_{44} .
\end{aligned}
$$

The obtained values of the compliance constants $S_{i j}$ of (B3) BN are listed and compared with other theoretical data [17] in Table I.

\subsection{Young's modulus, Poisson's ratio and Lamé constants}

There is considerable interest in the effect of mechanical stresses resulting from crystal growth and device processing on the behavior and reliability of semiconductor devices. Precise computation of such stresses requires 
knowledge of Young's modulus and Poisson's ratio, in particular for specific orientations within the crystallographic plane defining the surface of the semiconductor. Young's modulus $Y$ is not isotropic in cubic zinc-blende type crystals [41].

The modulus $Y$ for an arbitrary crystallographic direction $m$ can now be given by [37]:

$$
\begin{aligned}
\frac{1}{Y} & =S_{11}-2\left(S_{11}-S_{12}-\frac{1}{2} S_{44}\right) \\
& \times\left(m_{1}^{2} m_{2}^{2}+m_{2}^{2} m_{3}^{2}+m_{1}^{2} m_{3}^{2}\right),
\end{aligned}
$$

where the $S_{i j}$ values are the elastic compliance constants and the $m$ values are the direction cosines for $m$. Poisson's ratio $P$ also varies with orientation. If a longitudinal stress in the direction $m$ and the transverse strain along the orthogonal direction $n$ are under consideration, then the ratio $P$ can be given by [37]:

$$
\begin{aligned}
P & =\left[\left(S_{11}-S_{12}-\frac{1}{2} S_{44}\right)\left(m_{1}^{2} n_{1}^{2}+m_{2}^{2} n_{2}^{2}+m_{3}^{2} n_{3}^{2}\right)\right] \\
& /\left[S_{11}-2\left(S_{11}-S_{12}-\frac{1}{2} S_{44}\right)\right. \\
& \left.\times\left(m_{1}^{2} m_{2}^{2}+m_{2}^{2} m_{3}^{2}+m_{1}^{2} m_{3}^{2}\right)\right] .
\end{aligned}
$$

The modulus $Y$ for the direction of the cube axes $(\langle 100\rangle)$ is given by $Y=1 / S_{11}$. The ratio $P$, in this case, is written as $P=-S_{12} / S_{11}$. The variations of $Y$ and $P$ for directions within the important crystallographic planes $\{100\},\{110\}$, and $\{111\}$ are listed in Table II. It should be noted that the $Y$ and $P$ are invariant within the $\{111\}$ plane [42].

\section{TABLE II}

Mechanical parameters of (B3) BN: Young's modulus $Y$, Poisson's ratio $P$ and the Lamé constants $(\mu, \lambda)$ for directions within the important crystallographic planes $\{100\},\{110\}$ and $\{111\}$ at $p=0, T=0 \mathrm{~K}$.

\begin{tabular}{c|c|c|c|c|c}
\hline \hline Plane & Direction & $Y$ [Mbar] & $p$ & $\mu$ [Mbar] & $\lambda[$ Mbar] \\
\hline$\{100\}$ & $\langle 001\rangle$ & $7.402,7.46[17]$ & & & \\
& $\langle 011\rangle$ & $9.274,9.39[17]$ & & & \\
$\{110\}$ & $\langle 001\rangle$ & $7.402,7.46[17]$ & & & \\
& $\langle 111\rangle$ & $10.123,10.3[17]$ & & & \\
$\{111\}$ & & $9.274,9.39[17]$ & & & \\
$\{100\}$ & $m=\langle 010\rangle, n=\langle 001\rangle$ & & $0.189,0.187[17]$ & 3.113 & -0.146 \\
& $m=\langle 011\rangle, n=\langle 0 \overline{1} 1\rangle$ & & $-0.016,-0.024[17]$ & 4.712 & 1.891 \\
$\{110\}$ & $m=\langle 001\rangle, n=\langle 1 \overline{1} 0\rangle$ & & $0.189,0.187[17]$ & 3.113 & 0.831 \\
& $m=\langle 1 \overline{1} 1\rangle, n=\langle 1 \overline{12}\rangle$ & & $0.075,0.069[17]$ & 4.708 & 1.773 \\
\hline 111$\}$ & & & $0.153,0.149[17]$ & 4.022 & \\
\hline
\end{tabular}

[17] Calculated using $\left(S_{11}=0.137, S_{12}=-0.025, S_{44}=0.208\right) \mathrm{Mbar}^{-1}$ at $T=300 \mathrm{~K}$.

For cubic crystals, the Lamé constants $(\mu, \lambda)$, Young's modulus $Y$ and the Poisson ratio $P$ are related by [43]:

$$
\begin{aligned}
& \mu=Y /[2(1+P)], \\
& \lambda=P Y /[(1+P)(1-2 P)] .
\end{aligned}
$$

The variations of $\mu$ and $\lambda$ for directions within the important crystallographic planes $\{100\},\{110\}$ and $\{111\}$ are listed also in Table II. Unfortunately, there are no data available in the literature on these properties for this compound. Future experimental work or other theoretical calculations will contribute with more tests for the validating of our calculated results of these parameters.

\subsection{Sound velocity}

A perfect isotropic material is one for which isotropy factor $=1.0$. As seen in Table II, BN is not perfectly isotropic in its elastic properties. Sound velocities in this material are thus, strongly dependent on the propagation directions. If the crystal density $g$ and the stiffness con- stant $C_{i j}$ of a solid are known, one can calculate the bulk sound velocity $v$ (long-wavelength, nondispersive) from the following general relation [35, 37]:

$$
v=\left(C_{i j} / g\right)^{1 / 2} \text {. }
$$

In general, there are three types of wave motion for a given direction of propagation in the crystal, but only for a few special directions can the waves be classified as pure longitudinal or pure transverse. If we neglect nonlinear terms in the equation of motion, pure longitudinal sound waves may propagate in the [100], [110], and [111] directions. Pure transverse waves may propagate not only in these three directions, but also in the crystallographic directions [ $h k l](h=k ; l$ arbitrary) and [ $h k 0]$ ( $h$ and $k$ arbitrary).

We can see in Table III of Ref. [41] definition of sound velocity expressed in terms of these constants along the high-symmetry directions [100], [110] and [111] in cubic zinc-blende crystals. The obtained values of sound veloc- 
ities for major directions in the (B3) BN at zero-pressure are reported in Table III.

\section{TABLE III}

The sound velocities for major directions in the cubic, zinc-blende lattice of $\mathrm{BN}$, as controlled by the second-order elastic constants $C_{i j} .{ }^{a}$ Longitudinal acoustic waves, ${ }^{b}$ transverse acoustic waves.

\begin{tabular}{c|c|c}
\hline \hline $\begin{array}{c}\text { Propagation } \\
\text { direction }\end{array}$ & $\begin{array}{c}\text { Direction or plane } \\
\text { of polarization }\end{array}$ & $\begin{array}{c}\text { Sound velocity } \\
{\left[\times 10^{5} \mathrm{~cm} / \mathrm{s}\right]}\end{array}$ \\
\hline$[100]$ & {$[100]^{a}$} & $15.015,15.3[17]$ \\
{$[110]$} & $(100)^{b}$ & $11.597,11.7[17]$ \\
& {$[100]^{a}$} & $15.672,16.8[17]$ \\
& {$[001]^{b}$} & $11.597,11.7[17]$ \\
{$[111]$} & {$[1 \overline{1} 0]^{b}$} & $9.3113,9.5[17]$ \\
\multicolumn{2}{|c}{$[111]^{a}$} & $15.886,17.3[17]$ \\
\hline 17$]$ Calculated using $C_{11}=8.20, C_{12}=1.90, C_{44}=$ \\
4.80 Mbar and $g=3.8880 \mathrm{~g} / \mathrm{cm}^{3}$ at $T=300 \mathrm{~K}$.
\end{tabular}

\subsection{Calculation of Debye temperature}

Having calculated the bulk modulus $B$ and shear modulus $G$, one can calculate the Debye temperature, which is an important fundamental parameter closely related to many physical properties such as elastic constants, specific heat and melting temperature.

At low temperature the vibrational excitation arises solely from acoustic modes. Hence, at low temperatures the Debye temperature calculated from elastic constants is the same as that determined from specific heat measurements. One of the standard methods to calculate the Debye temperature $\left(\theta_{\mathrm{D}}\right)$ is from elastic constants data, since $\theta_{\mathrm{D}}$ may be estimated from the average sound velocity, $v_{m}$ by the following equation [29]:

$$
\theta_{\mathrm{D}}=\frac{h}{k_{\mathrm{B}}}(3 / 4 \pi V a)^{1 / 3} v_{m}
$$

where $h$ is the Planck constant, $k_{\mathrm{B}}$ - the Boltzmann constant and $V_{a}$ - the atomic volume. The average sound velocity is given by [29]:

$$
v_{m}=\left[\frac{1}{3}\left(\frac{2}{v_{t}^{3}}+\frac{1}{v_{l}^{3}}\right)\right]^{-1 / 3},
$$

where $v_{l}$ and $v_{t}$ are the longitudinal and transverse sound velocity obtained using the shear modulus $G$, the bulk modulus $B$ and the density $g$ from Navier's equation [29]:

$$
\begin{aligned}
& v_{l}=[(3 B+4 G) / 3 g]^{1 / 2}, \\
& v_{t}=(G / g)^{1 / 2} .
\end{aligned}
$$

The calculated sound velocity and Debye temperature as well as the density for (B3) BN are given in Table IV. To the best of our knowledge, there is a little [44-46] data available in the literature on these properties for this compound.

The Debye temperature calculated is in excellent agreement with data available in the literature [44-46],

\section{TABLE IV}

The longitudinal, transverse and average sound velocity $\left(v_{l}, v_{t}, v_{m}\right.$ in $\left.10^{3} \mathrm{~m} / \mathrm{s}\right)$ calculated from isotropic elastic modulus, and the Debye temperatures $\left(\theta_{\mathrm{D}}\right.$ in $\left.\mathrm{K}\right)$ derived from the average sound velocity for (B3) BN in comparison with data available in the literature [17, 44-46].

\begin{tabular}{c|l}
\hline \hline$v_{l}$ & $15.015,15.3[17]$ \\
$v_{t}$ & $9.3113,9.5[17]$ \\
$v_{m}$ & 10.266 \\
$\theta_{\mathrm{D}}$ & $1707.56,1613[44]$ at $300 \mathrm{~K}, 1730[45]$ at $70 \mathrm{~K}$, \\
& $1987[46]$ at $0 \mathrm{~K}$
\end{tabular}

which deviate from the measured [45] with $1.3 \%$. This marked result signified the good values obtained for the second-order elastic constants $C_{i j}$ and other mechanical parameters.

\section{Conclusions}

Employing PP-PW approach based on density functional theory, within the local density approximation, we studied the structural and elastic properties, sound velocity, and the Debye temperature and anisotropy effect on the mechanical parameters for boron phosphide in its structure zinc-blende phase leads to the conclusions to be summarized as follows:

1. The calculated structural properties of this compound are in good agreement with the available experimental and theoretical data reported in the literature.

2. The pressure dependence of the unit cell volume and relative unit cell volume parameters are calculated, the continuity in pressure--volume phase diagram indicate the absence of the structural phase transition from zinc-blende (B3) to rock salt (B1) up to pressure of 4 Mbar.

3. A numerical first-principles calculation of the elastic constants $\left(C_{11}, C_{12}\right.$, and $\left.C_{44}\right)$, the results obtained are also in good agreement with the available experimental and theoretical data reported in the literature.

4. The values obtained of the elastic constants was used to predict the anisotropy effect on the several mechanical parameters such as Young's modulus $Y$, Poisson's ratio $P$, Lamé constants $(\mu, \lambda)$ and the sound velocity $v$ for directions within the important crystallographic planes and it was used also to calculate the Debye temperature for (B3) BN compound. The result obtained of the Debye temperature is also in good agreement with the available experimental and theoretical data reported in the literature.

\section{References}

[1] O.A. Golikova, Phys. Status Solidi A 51, 11 (1979).

[2] R.M. Wentzcovitch, K.J. Chang, M.L. Cohen, Phys. Rev. B 34, 1071 (1986).

[3] Numerical Data and Functional Relationships in Science and Technology - Crystal and Solid State Physics, Vol. III of Landolt-Börnstein, Ed. O. Madelung, Springer, Berlin 1972. 
[4] R.H. Wentorf, J. Chem. Phys. 26, 956 (1957).

[5] C.A. Brookes, R.M. Hooper, W.A. Lambert, Philos. Mag. A 47, L9 (1983).

[6] E. Knittle, R.M. Wentzcovitch, R. Jeanloz, M.L. Cohen, Nature (London) 337, 349 (1989).

[7] Numerical Data and Functional Relationships in Science and Technology-Physics of Group IV Elements and III-V Compounds, Vol. 17, Landolt-Börnstein, Ed. O. Madelung, Springer, Berlin 1982.

[8] V.L. Solozhenko, D. Hausermann, M. Mezouar, M. Kunz, Appl. Phys. Lett. 72, 1691 (1998).

[9] G A. Slack, S.F. Bartram, J. Appl. Phys. 46, 89 (1975).

[10] P.R. Hernandez, M.G. Diaz, A. Munoz, Phys. Rev. B 51, 14705 (1995).

[11] V.A. Pesin, Sverktverd. Mater. 6, 5 (1980).

[12] N.E. Christensen, I. Gorczyca, Phys. Rev. B 50, 4397 (1994).

[13] R.M. Wentzcovitch, M.L. Cohen, P.K. Lam, Phys. Rev. B 36, 6058 (1987).

[14] W. Sekkal, B. Bouhafs, H. Aourag, M. Certier, J. Phys. Condens. Matter 10, 4975 (1998).

[15] A. Zaoui, F. El Haj Hassan, J. Phys. Condens. Matter 13, 253 (2001).

[16] M.H. Manghnani, in: Proc. 5 NIRIM Int. Symp. Advanced Materials (ISAM '98) Tsukuba, Tsukuba 1998, p. 73 .

[17] S. Adachi, Handbook on Physical Properties of Semiconductors, Vol. 2, Kluwer Academic Publishers, Boston 2004.

[18] S.Yu. Davydov, S.K. Tikhonov, Semiconductors 30, 683 (1996).

[19] K. Kim, W.R.L. Lambrecht, B. Segall, Phys. Rev. B 53, 16310 (1996).

[20] T. Azuhata, T. Sota, K. Suzuki, J. Phys. Condens. Matter 8, 3111 (1996).

[21] K. Karch, E. Bechstedt, Phys. Rev. B 56, 7404 (1997).

[22] K. Shimada, T. Sota, K. Suzuki, J. Appl. Phys. 84, 4951 (1998).

[23] M. Grimsditch, E.S. Zouboulis, J. Appl. Phys. 76, 832 (1994).
[24] S.Q. Wang, H.Q. Ye, Phys. Status Solidi B 240, 45 (2003).

[25] W. Kohn, L.J. Sham, Phys. Rev. 140, A1133 (1965).

[26] The ABINIT computer code. Available online at http: //www . abinit.org .

[27] J.P. Perdew, K. Burke, M. Ernzerhof, Phys. Rev. Lett. 77, 3865 (1996 appendix).

[28] N. Troullier, J.L. Martins, Phys. Rev. B 43, 1993 (1991).

[29] A. Bouhemadou, Braz. J. Phys. 40, 52 (2010).

[30] H.J. Monkhorst, J.D. Pack, Phys. Rev. B 13, 5189 (1976).

[31] F. Murnaghan, Proc. Nat. Acad. Sci. USA 30, 244 (1944).

[32] C.G. Broyden, J. Inst. Math. Appl. 6, 222 (1970)

[33] F. Birch, J.J. Geophys. Res. 83, 1257 (1978).

[34] F. El Haj Hassan, H Akbarzadeh, M. Zoaeter, J. Phys., Condens. Matter 16, 293 (2004).

[35] C. Kittel, Introduction to Solid State Physics, Wiley, New York 1953.

[36] O.H. Nielsen, R.M. Martin, Phys. Rev. B 32, 3792 (1985).

[37] S. Adachi, Properties of Group-IV, III-V and II-VI Semiconductors, Wiley, England 2005.

[38] G.R. Barsch, Phys. Status Solidi 19, 129 (1967).

[39] W.A. Harrison, Electronic Structure and Properties of Solids, Dover, New York 1989.

[40] J.F. Nye, Physical Properties of Crystals, Clarendon, Oxford 1972.

[41] W.A. Brantley, J. Appl. Phys. 44, 534 (1973).

[42] T.D. Riney, J. Appl. Phys. 32, 454 (1961).

[43] O.O. Anderson, J. Phys. Chem Solids 24, 909 (1963).

[44] T. Atake, S. Takai, A. Honda, Y. Saito, K. Saito, Rep. Res. Lab. Eng. Mater. Tokyo Inst. Technol. 16, 15 (1991).

[45] Z.I. Kolupayeva, M.Ya. Fuks, Z.I. Gladkikh, A.V. Arinkin, S.V. Malikhin, J. Less-Common Met. 117, 259 (1986).

[46] H. Siethoff, K. Ahlborn, Phys. Status Solidi B $\mathbf{1 9 0}$, $179(1995)$. 